\title{
¿Cuánto tiempo me llevará salir de todo esto?
}

\section{Una aproximación etnográfica a la aplicación de "medidas alternativas" sobre jóvenes en Santiago del Estero}

Federico Medina ${ }^{1}$

1 Indes (CONICET-UNSE), Santiago del Estero, Argentina

(iD) https://orcid.org/oooo-0003-3969-8461

Correo electrónico: federicomedinas@gmail.com
Recibido:

agosto de 2020

Aceptado:

diciembre de 2020

doi: $10.34096 /$ cas.i53.8480

\section{Resumen}

En este artículo he adoptado una perspectiva antropológica para describir y analizar los usos locales y los sentidos diversos asociados a la implementación de "medidas alternativas" sobre jóvenes, por parte de la Oficina de Medidas Alternativas y Sustitutivas del Poder Judicial de Santiago del Estero, provincia argentina situada en la región Noroeste. Para ello, recurro a la reconstrucción de diversas situaciones etnográficas acontecidas en esa dependencia judicial en el marco de una investigación de doctorado, buscando indagar en las perspectivas tanto de los jóvenes que transitan por esa oficina como de los actores institucionales que se relacionan con ellos durante el proceso de ejecución de estas medidas. El argumento central del trabajo apunta a iluminar la dimensión cotidiana del funcionamiento de esta dependencia judicial, poniendo en evidencia que la utilización de medidas alternativas adquiere formas domésticas y usos locales particulares que se sostienen sobre los mismos tópicos que dieron fundamento a las medidas tutelares tradicionales.

\section{How long will it take me to get out of all this? An ethnographic approach to the application of "alternative measures" on young people in Santiago del Estero}

\author{
Abstract \\ This article adopts an anthropological perspective to describe and analyze the local uses \\ and diverse meanings associated with the implementation of "alternative measures" \\ on young people, by the Office of Alternative and Substitute Measures of the Judicial \\ Power of Santiago del Estero, an Argentine province located in the Northwest region.
}

\section{Palabras clave}

Medidas alternativas; Usos; Procesos penales; Jóvenes; Etnografia

\section{Key words}

Alternative measures; Uses; Criminal proceedings; Youth; Ethnography 
To do this, I resort to the reconstruction of various ethnographic situations that occurred in that judicial unit within the framework of my doctoral research, seeking to investigate the perspectives of both the young people who pass through that office and the institutional actors who interact with them during the process of executing these measures. The central argument aims to illuminate the daily dimension of this judicial unit's operation, showing that the use of alternative measures takes on domestic forms and particular local uses that are sustained on the same topics upon which the traditional.

\section{Quanto tempo vou levar para sair de tudo isso? Uma abordagem etnográfica para a aplicação de "medidas alternativas" na juventu- de de Santiago del Estero}

\section{Resumo}

Palavras-chave

Medidas alternativas; Usa; Processos criminais; Juventude; Etnografia
Neste artigo optei por uma perspectiva antropológica para descrever e analisar os usos locais e os diversos significados associados à implementação de "medidas alternativas" entre os jovens implementadas pelo Escritório de Medidas Alternativas e Substitutas do Poder Judiciário de Santiago del Estero, província Argentina situada na região Noroeste. Com esse intuito, faço a reconstrução de várias situações etnográficas ocorridas naquela unidade judicial no âmbito de uma investigação de doutorado, procurando investigar as perspectivas tanto dos jovens que passam por aquele escritorio como dos atores institucionais que com eles se relacionam durante os processos de execução dessas medidas. $\mathrm{O}$ argumento central do trabalho visa iluminar a dimensão habitual do funcionamento desta unidade judicial, evidenciando que o uso de medidas alternativas apresenta formas domésticas e usos locais particulares que se sustentam nas mesmas temáticas que fundamentaram as medidas tutelares tradicionais.

\section{Introducción}

“¿Cuánto tiempo me llevará salir de todo esto?”, preguntó Juan, con la voz entrecortada, ansiosa y expectante, a punto de entrevistarse con la trabajadora social de la Oficina de Medidas Alternativas y Sustitutivas. Se trata de una pregunta que se repite una y otra vez y atraviesa las experiencias de jóvenes en el campo penal juvenil a nivel local. Se reproduce de diferentes maneras, adquiere distintos tonos y cobra determinados sentidos en los escenarios que marcan las trayectorias institucionales juveniles. En esta situación, el interrogante se enmarca en el ámbito de una oficina judicial que interviene con posterioridad a las audiencias judiciales donde, llegado el caso, se dispone la salida en libertad de jóvenes que atravesaban una situación de encierro luego de haber sido denunciados por la comisión de delitos.

En este artículo voy a analizar los usos locales y sentidos diversos que adquiere la implementación de "medidas alternativas" sobre jóvenes por parte de la Oficina de Medidas Alternativas y Sustitutivas del Poder Judicial de Santiago del Estero (en adelante, OMAS). Este estudio se inscribe en una línea de trabajos que, desde una perspectiva socioantropológica, han indagado sobre el funcionamiento de mecanismos denominados restaurativos en el ámbito judicial (Nader, 1994; Schuch, 2008; Mello y Baptista, 2011; Godoy, 2016; Matta y Godoy, 2016; Medan, 2016; Medan y Graziano, 2019; Villalta y Llobet, 2019; Villalta y Graziano, 2020, entre otros). La exploración se sitúa en esta dependencia judicial, creada recientemente con la reforma procesal penal 
a nivel local y encargada de supervisar y controlar todo tipo de "medidas alternativas" en causas judiciales que involucran tanto a jóvenes como a adultos.

Para hacerlo, voy a reconstruir diferentes situaciones etnográficas de las que he participado durante el trabajo de campo, realizado desde 2016 a 2018, para mi investigación doctoral. Esta investigación ha implicado la realización tanto de entrevistas abiertas o no directivas como observación participante durante el horario de funcionamiento cotidiano (matutino) de esta oficina judicial; así como numerosas conversaciones con activistas, funcionarios judiciales e instancias de acompañamiento y asesoramiento con los jóvenes y sus familias. El análisis de este material empírico será complementado con algunas conclusiones a las que arribaron diferentes investigaciones que fueron elaboradas a demanda de organismos supranacionales (Fondo de las Naciones Unidas para la Infancia/UNICEF, 2018; Banco Interamericano de Desarrollo/BID, 2021) en las que tuve la oportunidad de participar.

Esta investigación ha sido posible en la medida en que me he involucrado con los jóvenes y sus familias tras construir una relación colaborativa con la organización "La Poderosa", con quienes me contacté, desde mi condición de abogado, a partir de asesorar y representar legalmente a muchos de estos jóvenes en el ámbito judicial. Esta circunstancia marcó las condiciones de factibilidad de esta investigación, ya que pude aprovechar un cúmulo de vínculos previos con actores judiciales de diferente rango que me facilitaron la posibilidad de contar con instancias privilegiadas de observación participante, acceder a documentos judiciales, así como mantener distintas entrevistas y conversaciones informales con muchos de ellos. Simultáneamente, esto me demandó constantes ejercicios de reflexividad y distanciamiento respecto del objeto de investigación que construí, lo que ha generado notables desafíos tanto epistemológicos como metodológicos que pude analizar con mayor profundidad en otro trabajo (Medina, 2021).

En este artículo, la exposición será guiada a partir de la presentación de algunas situaciones vinculadas a una causa judicial por el delito de "hurto", seguida a Juan, un joven de 17 años que cuenta con numerosas experiencias de tránsito por la burocracia policial y judicial. Para ello, voy a recuperar las perspectivas de los jóvenes, de sus familiares y amigos, respecto del funcionamiento de la OMAS en relación con delitos "contra la propiedad" imputados a jóvenes incluidos en el régimen penal juvenil. Luego expondré una situación de visita a esta oficina judicial, en la que tuve oportunidad de acompañar a Juan, quien debió de asistir una y otra vez para dar cuenta del cumplimiento de las medidas "alternativas" que le habían sido impuestas en el marco de la causa a la que aludí.

Me interesa problematizar la tantas veces enunciada condición de "alternativa" de la medida judicial, retomando la perspectiva propuesta por Mello y Baptista (2011), así como discutir el carácter "restaurativo" de estas medidas que vendrían a reducir la "punitividad" del sistema judicial penal. El argumento que presento busca evidenciar cómo el funcionamiento de esta dependencia judicial oscila entre el control y el seguimiento de las habituales medidas alternativas (por ej., "probation") y la evaluación y el control de pautas de conducta (como las medidas "tutelares" típicas) impuestas por los jueces a los jóvenes como condición para salir en libertad. Así, intentaré iluminar cómo las reformas inspiradas en la idea de "justicia restaurativa" y "mecanismos alternativos de resolución de conflictos" se imbrican con una trama de relaciones institucionales preexistentes que funciona con lógicas y estrategias inspiradas en una tradición jurídica vinculada a la "justicia de menores", a la vez que procura adaptarse, en esa clave de justicia restaurativa, a la generación de concepciones y lógicas diferenciadas de intervención, lo que también ha sido caracterizado como un proceso de "configuración recíproca" (Villalta, Graziano, Gesteira, Grinberg, Fernández Tuñón y Pérez Álvarez, 2017). 


\section{¿Un escenario judicial “diferente”?}

De acuerdo con su reglamento de creación y funcionamiento, esta oficina judicial tiene un carácter "fundamental" en tanto "auxiliar" del sistema judicial, e interviene en el control y supervisión de todo tipo de medidas impuestas por los juzgados de garantías, ya se trate de causas de adultos como de jóvenes incluidos dentro del régimen penal de la minoridad.

Desde las perspectivas de los jóvenes, esta dependencia es percibida como "otra" oficina judicial. En más de una ocasión, escuché decir que se trata de "algo diferente a tener que ir a declarar frente a un juez", en el sentido de que se trata de una experiencia distinta a la de atravesar por una audiencia en la que los jóvenes se enfrentan a las acusaciones construidas entre la policía y las fiscalías y en cuyas escenas la figura del juez luce como preponderante. Ninguno de estos agentes institucionales se encuentra presente en esta dependencia judicial. Y en esa diferenciación acerca de la percepción sobre el mundo judicial, también se trama el vínculo que sostienen con las trabajadoras sociales de la OMAS con quienes se entrevistan. Estas profesionales encarnan a un agente estatal ubicado en la intermediación entre el universo de quienes son administradores y el de quienes son administrados en la justicia penal, como ya ha sido señalado en otras investigaciones que han explorado el desempeño de figuras burocráticas semejantes (Lugones, 2012; Graziano, 2017). A su vez, esta categorización como "otro" espacio judicial también se vincula con la situación en la que les toca a los jóvenes presentarse en esta oficina, generalmente en condición de libertad y acompañados de algún familiar. Esto abona la idea de que se trata de un escenario institucional más "amigable", en comparación con las audiencias en el juzgado y otros actos procesales que cumplen con anterioridad y a los que por lo general llegan esposados y escoltados por agentes policiales.

Mirian, madre de Juan, me contaba que en las ocasiones en las que debió presentarse a la OMAS para dar explicaciones e informar sobre "el problema de su hijo", comentaba todo lo que le habían informado sobre el tratamiento por adicciones que este tenía que hacer, lo que le decían en la escuela nocturna respecto a la situación en que se encontraba, cómo andaba Juan en el barrio, etc. Tenía la posibilidad de explayarse y de contar "todo lo que pasa sin tener que esperar a que un juez nos deje hablar" (Registro de diario de campo, Entrevista con Mirian, Santiago del Estero, mayo de 2018).

Por su parte, el seguimiento y supervisión de las medidas ordenadas por los jueces ubica a la OMAS con respecto a un conjunto de instituciones de variada índole, lo que permite identificar un campo compuesto por una gran cantidad de actores, posiciones y relaciones sociales entabladas a propósito del cumplimiento de medidas socioeducativas por parte de los jóvenes. Este amplio y heterogéneo espectro de vínculos interinstitucionales evidencia el carácter complejo y multidimensional que presenta la actuación estatal en este campo. Así, iluminar al Estado desde la idea de su deshomogeneización buscando eludir su reificación, en el sentido que ha sido reflexionado desde la sociología política por Abrams (2000), así como desde la antropología política por Das y Poole (2008), permite identificar la tensa convivencia de dos aspectos centrales en el campo discursivo de los derechos de la infancia y la adolescencia. Por un lado, la administración del castigo frente a la conflictividad penal juvenil; por el otro, la protección y "restitución de derechos" de los jóvenes verificada en las acciones estatales encaminadas al cuidado e inclusión social, lo que también se señaló para visibilizar cómo estas combinan tanto asistencia como represión (Villalta, 2013).

Para hacer su tarea, las trabajadoras sociales de la oficina deben ponerse en contacto con instituciones provinciales y nacionales del ámbito del "consumo problemático" de estupefacientes (Dirección General de Abordaje Integral de las Adicciones de la Provincia -DIGAIA- y la Coordinación Nacional de Políticas contra las Adicciones 
-SEDRONAR-), con organismos provinciales de protección de derechos de la infancia y adolescencia (Subsecretaría de Niñez, Adolescencia y Familia, por ejemplo), establecimientos escolares donde los jóvenes deben retomar sus estudios, la policía, actores y organizaciones de la sociedad civil y barriales, etc. Así, sus intervenciones están directamente ligadas a una serie de recursos (tales como programas de reinserción escolar o el ingreso a instituciones públicas o privadas donde los jóvenes, además de desintoxicarse, puedan afrontar un tratamiento de un cuadro de adicciones, etc.), que se presentan con muy baja disponibilidad en la provincia, lo que depara grandes dificultades en el cumplimiento de las medidas impuestas por los jueces.

Esta dimensión de relacionamiento, entonces, posiciona a esta oficina judicial en contacto con un universo muy amplio de burocracias estatales y organizaciones de la sociedad civil que funcionan con lógicas, tramas político-institucionales y formas de trabajo diversas.

Este amplio abanico de relaciones e interacciones, tanto interinstitucionales como de trato directo con las madres y los jóvenes, también abona a la idea de que esta oficina judicial se concibe como "otra". Se trata de una "otredad" construida a partir de un sentido que asocia a la justicia con un ámbito de interacciones reguladas por solemnidades, reglas y rituales (Garapon, 1997) que guardan poca semejanza con las formas de intervención típicas de la OMAS. Es así como la perspectiva etnográfica adoptada ha permitido echar luz sobre

espacios e interacciones del poder judicial en los que la distancia, el hermetismo y formalismo del discurso y de las prácticas se quiebra en puntos de encuentro entre las personas de carne y hueso que "circulan por" y trabajan en esos espacios. (Eilbaum, 2017, p. 11-12)

Como lo indiqué, en tanto puente entre el universo de administradores y el de administrados del mundo judicial o bien entre el formalismo del juez u "orden legal" (Mello y Baptista, 2011) y la realidad social de los jóvenes, en este escenario cobra relevancia la figura de las trabajadoras sociales que se entrevistan periódicamente con los jóvenes para evaluar/examinar/controlar el cumplimiento de las medidas que previamente fueron ordenadas por los jueces. El análisis del rol de estas operadoras judiciales permitió problematizar una caracterización muy extendida entre actores del campo jurídico (Bourdieu y Teubner, 1976) que atribuyen a la OMAS la condición de dependencia de tipo meramente "administrativa" o como ámbito judicial de menor jerarquía en donde supuestamente no se toman decisiones sobre la culpabilidad o inocencia de los jóvenes.

Desde la perspectiva de los jóvenes y de sus madres, así como también de activistas de la organización "La Poderosa" que trabajan con ellos, esta oficina se percibe como un ámbito institucional permeable a muchas de las demandas de contención que reclaman necesarias, lo que permitiría atribuirle cierto rasgo "restaurativo" en el sentido que propone Medan (2016) al referirse al potencial que presenta la justicia restaurativa para recomponer las relaciones de los jóvenes con las instituciones judiciales. Algunos de los jóvenes aseguran que las trabajadoras sociales con quienes se entrevistan tienen una mayor sensibilidad y capacidad de comprensión de "todo lo que nos pasa", a diferencia de los jueces, a los que estiman como más "cerrados". Con algunos matices, afirman que allí pueden encontrar una escucha activa a sus demandas, es decir, un espacio en el que es posible ser "escuchados y no simplemente castigados" (Medan, 2016, p. 86). Tanto Juan como los jóvenes con quienes he conversado me han comentado que, si bien algunas veces les han tocado trabajadoras "no tan piolas", en la mayoría de los casos se encontraron con personas con quienes pudieron conversar $\mathrm{y}$ contarles sus problemas. 
Rodrigo, activista de "La Poderosa" que suele acompañar a Juan y otros jóvenes de su barrio cuando tienen que ir a la OMAS, me decía:

Mira, aquí hay profesionales que entienden que es todo lo que pasa y cómo son las cosas en los barrios. Son trabajadores sociales que tienen otra mentalidad. Yo entiendo que hay todo tipo de jueces y hay algunos que tienen cero compromiso y quienes alguito buscan entender todo el problema. Con los que no quieren entender y toman todo lo que hace y dice la policía esto es difícil de remontar, cuando ellos deciden sobre los jóvenes las trabajadoras de la OMAS hacen lo que pueden, porque están obligadas. Pero cuando se puede, se comprometen con otro tipo de tratamiento para los chicos. Y los chicos lo saben a esto, porque, a diferencia de los jueces y fiscales, y en algunos casos hasta de los propios defensores oficiales de los chicos, las trabajadoras sociales los escuchan y los miran a la cara. Ellos se sienten mejor ahí adentro y si no andan pasados de la droga, no te hacen problemas para venir aquí. (Registro de diario de campo, entrevista con Rodrigo, Tribunales, Santiago del Estero, mayo de 2018)

\section{Dinámica de una visita a la OMAS. Esperar afuera}

Eran aproximadamente las nueve de la mañana cuando llegué a la casa de Juan, ubicada en un barrio popular de la ciudad de Santiago del Estero. Allí me esperaba el joven junto a su madre. Habían pasado dos días desde que la jueza de la causa había dispuesto su liberación, luego de pasar varias jornadas detenido en una comisaría. A los pocos minutos, llegó también Rodrigo. Nos habíamos reunido con el propósito de pensar cómo "sostener" el cumplimiento de las "medidas alternativas" que pesaban sobre Juan para estar a la altura de las expectativas institucionales de "normalización”, en tanto estas medidas no solo buscan "prevenir la violación de las leyes sino de inculcar normas y actitudes específicas” (Garland, 2018, p. 333), esperando que los jóvenes se responsabilicen por sus actos y gestionen con eficacia la gran cantidad de amenazas que se presentan a la hora de cumplir con ellas.

Las salidas en libertad y la obligación de cumplir con las "medidas alternativas" en la OMAS activan todo un complejo entramado de interacciones en el barrio donde vive Juan, así como en las instituciones de salud y educativas adonde los jóvenes deben concurrir para alcanzar su correcto cumplimiento. Cuando vuelven a sus casas, perciben que, fuera de sus familiares y algún que otro vecino con quien que mantienen buenas relaciones, en general nadie parece esperarlos. Ni aquel vecino que reclama por tal o cual objeto robado o les atribuye que son el "problema de la inseguridad en el barrio"; ni la policía que puede perseguirlos o detenerlos ni bien llegan; ni tampoco el "transa" (vendedor minorista de droga) que puede advertir en esto una oportunidad de cobrarse una deuda que quedó pendiente cuando el joven fue detenido.

El hecho de reinsertarse en el barrio implica, según sus percepciones, abocarse a generar diferentes mecanismos de autocuidado frente a estas amenazas concretas, así como lidiar con los juicios morales que pesan sobre sus conductas, lo que supone gestionar el riesgo que la "responsabilización” por sus actos les demanda.

Esta caracterización de la situación de regreso al barrio permite problematizar la concepción tan extendida que idealiza lo "comunitario" como escenario de cuidado y posibilitador de la "reinserción” del joven, en línea con lo que plantea Medan (2019) al llamar la atención sobre cómo estos abordajes invisibilizan el carácter amenazante de la comunidad o del barrio para los jóvenes. 
En la situación narrada, estas cuestiones percibidas como "desafíos" conforman el "temario" habitual de cada salida en libertad de un joven luego de un período de encierro y marcan el preludio de una primera visita a la OMAS. Así, luego de conversar detenidamente por casi una hora sobre estos temas, nos trasladamos hasta Tribunales, Juan, Rodrigo y yo. Durante el trayecto, Juan destacaba que no le generaba "tanto problema" ir a la OMAS porque lo hacía estando en libertad, y por ende, no llegaba "ni esposado ni maltratado por los policías que lo llevaban en un móvil". Presentarse nuevamente en Tribunales, ya sin la presencia policial, implicaba llegar con otro ánimo.

Nos habíamos organizado de manera apresurada con la expectativa de "llegar a tiempo" dentro del horario programado, impulsados por la rigidez con la que son concebidos los plazos temporales en el mundo judicial. Había que "hacer bien las cosas", según lo había ordenado la jueza al disponer las medidas en la audiencia previa.

Cuando llegamos habían pasado unos pocos minutos de las diez de la mañana. La oficina se encuentra en un largo pasillo que conecta los dos brazos principales del edificio judicial y cuenta con una puerta de ingreso y una ventanilla de mesa de entradas. A lo largo de ese pasillo, se ubican unos bancos en donde generalmente se espera para ingresar al interior de la oficina, una vez que los jóvenes son convocados para las entrevistas.

El horario previsto para la entrevista con la trabajadora social era a las 10:30. Nos acercamos a la mesa de entradas e informamos que ya estábamos presentes a la espera de que llamaran a Juan. Tan solo un puñado de situaciones fue suficiente para constatar que en la mayoría de los casos ese horario no se cumple con exactitud, por lo tanto, hay que esperar en el pasillo hasta que llamen al joven por su apellido.

En ese pasillo, había aproximadamente 20 personas esperando a ser atendidas. En ese primer acercamiento a este "pasillo de espera", fue posible observar con facilidad cómo se encontraban esperando por ingresar a las entrevistas muchos jóvenes pertenecientes a barrios populares de gran vulnerabilidad socioeconómica. La antesala a la entrevista duró alrededor de una hora y media, y fue el escenario para los más diversos intercambios de palabras entre Juan y un gran número de amigos y conocidos del barrio con quienes se encontró espontáneamente en el lugar.

El sistema de turnos para esperar por el ingreso a la OMAS se organiza no ya siguiendo la temporalidad rígida del "tiempo legal", como señala Tiscornia (2008), sino más bien de acuerdo con lo que ella denomina "tiempos judiciales", los cuales parecen determinarse "por el lugar estructural que diferentes actores ocupan durante el proceso y, en él, por quien se considera investido para ejercer el poder del 'tiempo oportuno': el juez" (Tiscornia, 2008, p. 137). Y si bien aquí "quien hace esperar" no es el juez sino los empleados administrativos de la oficina, quienes van definiendo uno a uno los ingresos para realizar las entrevistas, lo cierto es que el hecho de "hacerlos esperar" -como modo de ejercicio de poder (Auyero, 2012)- involucra también actualizar una y otra vez el "lugar estructural" que ocupan los jóvenes dentro de un proceso penal.

Mientras tanto, en ese "estar esperando" afuera, acontecen múltiples interacciones que, a priori, no tienen los límites de comportamiento que los jóvenes deben cumplir una vez que se entrevistan con una trabajadora social en el interior de la OMAS. Allí, he observado diferentes situaciones de socialización entre ellos, algunas, marcadas por la tensión a partir de reproches o amenazas de "ajustes de cuentas" por determinados hechos a los que solo se refieren de manera encriptada. En esos casos, la aparición de las trabajadoras sociales que cada tanto salen por la puerta de ingreso para llamarlos indicándoles su turno para ingresar, hace posible que esa tensión no se incremente en demasía: "ya te voy a encontrar allá a vos, sabes que aquí no te puede pasar nada, pero ya nos vamos a encontrar allá, fue habitual escuchar en dichas conversaciones. 
Mariana, trabajadora social entrevistada, me relataba que tanto ella como sus compañeras advertían que los jóvenes eran "unos afuera y otros cuando vienen aquí al box". Alli "afuera", señalaba, "se manejan con cierta libertad y hasta hubo quienes nos hacían gestos feos, pero cuando ingresaron se comportaron muy bien" (Registro de diario de campo, entrevista con Mariana, Tribunales, Santiago del Estero, mayo de 2018).

También he conversado con otra trabajadora social de la oficina, Paula, quien reforzaba esta cuestión. Mientras que "afuera" podían "pasarse de la raya", adentro debían "comportarse mejor". Le pregunté cómo significaba su condición de agente judicial que sostiene una posición de autoridad con los jóvenes que van a "rendir cuentas". Respondió relativizando el lugar de autoridad frente a ellos, restándole valor a ese rol, asegurando que ellas se proponen "generar otro de tipo de relación con los chicos, más cercana, conversando sobre su vida y sus deseos" (Registro de diario de campo, entrevista con Paula, Tribunales, Santiago del Estero, mayo de 2018).

Así, la dinámica de la espera en esa oficina se configura como un necesario paso burocrático en el que los jóvenes van ingresando a medida que una persona sale de esa puerta y grita con fuerza su nombre y apellido. Todos podíamos tomar conocimiento de quiénes entraban a las entrevistas, ya que los nombres se escuchaban por todo el largo pasillo y resonaban una y otra vez hasta que la persona se hacía presente en la puerta e ingresaba. De este modo, como ya he señalado, esta situación de espera actualiza la posición que ocupan los jóvenes de la trama de relaciones de este campo institucional. Quien que debe cumplir con la espera queda sujeto a ese ejercicio de poder y en ese espacio de tiempo acontecen esas experiencias diferenciales con relación a las esperas.

\section{Rendir cuentas adentro}

En el interior de la OMAS, los jóvenes se dirigen hacia las pequeñas oficinas dispuestas para las entrevistas individuales. Allí, tienen que dar cuenta del cumplimiento de las medidas. Tal como mencioné, los jueces que disponen las medidas son "jueces de adultos" que tienen también competencia en las causas penales de adolescentes, en tanto no existe en el orden local un juzgado penal específico de competencia en el ámbito juvenil. Y aquí la particularidad radica en que, tratándose de delitos "contra la propiedad" y otras figuras penales que no prevén penas de suma gravedad, es habitual que los jueces se limiten a disponer las medidas. Tras ello, no controlan su ejecución, ya que no se les otorga intervención en esta etapa, por lo que esto queda a cargo exclusivamente de la OMAS.

Estas medidas, que en ocasiones son denominadas con el mote de "medidas alternativas a cumplir en la OMAS" o, en otras, indistintamente con la expresión clásica de "tratamiento tutelar en el marco del régimen penal juvenil vigente", buscan impactar sobre sus entornos comunitarios, ya que actúan sobre las tramas sociales en las que se desarrollan sus vidas: familia, barrio, escuela e incluso, en algunos casos, en sus ámbitos laborales. Por lo general, concretamente consisten en: recuperar la escolaridad, conseguir un trabajo, iniciar tratamiento por adicciones y acreditar todas estas medidas con determinada periodicidad ante la OMAS.

Retomando la microescena que me interesa aquí analizar, luego de transcurrida una hora de espera, llamaron a Juan para que ingresara a la oficina. Una vez adentro, fue conducido hacia un pequeño "box" donde se realizaría la entrevista con los profesionales que seguían su causa. Esta entrevista tuvo un desarrollo que se repite en la mayoría de los casos relevados: la profesional abre el encuentro señalando las medidas que el joven tiene que cumplir y subraya con especial énfasis en el tratamiento por adicciones. Para cumplirlo, le indicó a Juan que le iba a entregar un "papelito" y que con eso 
tenía que ir a la DIGAIA. Una vez allí, esta dependencia sería la que se ocuparía de su tratamiento. Cada quince días tendría que presentarse nuevamente en la OMAS y traer "el papelito de la DIGAIA que indique que está haciendo el tratamiento"; lo que evidencia el valor que tiene el comprobante más allá de que el régimen indicado cumpla o no con los efectos que se persiguen al hacerlo. Luego de esto, Juan preguntó cuánto tiempo más tenía que ir a la OMAS. A lo que la trabajadora social le respondió que eso iba a depender de cómo avanzaba con el cumplimiento de las medidas y que, si "andaba bien", podía venir solo una vez al mes. Al finalizar la entrevista, se despidió con una advertencia: "si no cumple, él ya sabe que va a pasar" (Registro de diario de campo, entrevista con Juan, Tribunales, mayo de 2018).

Este tipo de diálogos entre operadoras judiciales y jóvenes se reproduce una y otra vez. En algunos casos, con el paso del tiempo y dado el constante incumplimiento de las medidas por parte de aquellos, las conversaciones adquieren mayor tensión. Las trabajadoras sociales, orientadas por ese tipo de intervención que, como señala Donzelot (1977), busca centrarse en la comprensión de las situaciones personales más que en la sanción penal, reclaman por las medidas e intentan explorar las razones por las cuales no pueden ir a la escuela o no logran sostener el tratamiento por adicciones. Los jóvenes en varias ocasiones se presentan a dar respuesta, pero en muchas otras se ausentan a las entrevistas programadas.

En la mayoría de los casos, de una forma u otra, aparece la cuestión de las consecuencias del incumplimiento, generalmente mediante la expresión: "si no cumples, ya sabes qué va a pasar", en tanto recurso disponible para forzar, obligar o comprometer a los jóvenes. Juan comentaba que había en la OMAS quienes se lo "decían bien y otras no tanto, pero que igual yo ya sabía qué iba a pasar si no cumplía con las medidas". Esta amenaza de la penalización también evidencia el carácter sancionatorio que adquiere la "medida alternativa" en este contexto y funciona como un modo de "penalización sin custodia" (Amorim, Kant de Lima y Burgos, 2003, p. 55).

En el interior de la oficina individual donde se realizan las entrevistas, la rendición de cuentas se tematiza alrededor de la recuperación de las obligaciones en la escuela, de la reinserción en redes barriales que apuntan a construirse como espacios de contención, de problemas familiares, de tratamientos por adicciones y sus vicisitudes. Los sentidos son muy diversos. Allí adentro, cobran un valor particular las performances juveniles frente a las trabajadoras sociales, ya que los jóvenes deben exhibir un proceso de transformación en curso. Resulta sugerente pensar estas situaciones a partir de la idea de "dramatización de la transformación" propuesta por Graziano (2017): se trata de una oportunidad en donde se disponen a "mostrar un cambio" y persuadir de su "transformación". Justamente la división entre un "afuera" donde se espera y un "adentro" donde acontece la entrevista en sí, busca reflejar dos modos de performance juvenil discernibles entre sí.

Toda esta forma de interacción genera una cercanía y conocimiento de la vida de los jóvenes en línea con lo que Garland denominó como la "extensión del poder judicial", para dar cuenta de cómo la intervención penal se orienta al "ingreso en la vida y hogares” de los jóvenes (Garlan, 2018 p. 333), ya que busca construir, por una parte, alianzas con sus familias, quienes a su vez son percibidas como "parte de la solución, como agente de control social en las medidas en libertad" (Tenenbaum, 2018, p. 170). Pero por otra parte, estas medidas se sustentan también en la evaluación sobre esas familias, como requisito para la aplicación y cumplimiento de dichas medidas. Esto permite apreciar cómo el "ingreso en la vida y hogares" de los jóvenes se trama tanto en términos de alianza/cooperación como de vigilancia/evaluación sobre sus familias y entornos comunitarios. 


\section{La dimensión temporal de las medidas "alternativas"}

Durante el desarrollo de las entrevistas, una preocupación central parece atravesar el estado emocional de los jóvenes. Se trata del interrogante que construyen como: “¿Cuánto tiempo dice usted que me va a llevar todo esto?". En el "todo esto" también se incluye esa dimensión amenazante de la comunidad y la percepción subjetiva de que las posibilidades de recuperación luego de un período de encierro son muy limitadas.

El tiempo que llevará "salir de todo esto" está marcado por la temporalidad de un proceso judicial y no por los tiempos personales que pudiera demandar, por caso, la recuperación de una situación de adicción. Estos "tiempos judiciales", siguiendo a Tiscornia, implican que este ámbito define sus "acontecimientos significativos para un grupo, y cada grupo tiene sus propios puntos de referencia en función del espacio social en que organiza la vida social” (Tiscornia, 2008, p. 133). Por su parte, los "tiempos personales" que cada subjetividad demanda para salir de una determinada situación complicada también están fuertemente atravesados por la discrecionalidad judicial en la evaluación del tiempo, a la que alude Tedesco (2017) cuando detiene su atención sobre las diferentes temporalidades a las que están sujetos los jóvenes durante las experiencias de encierro en los centros juveniles de detención de la ciudad de Córdoba.

Resulta sugerente analizar cuáles son los hechos que se consideran como "acontecimientos significativos" para quienes deben evaluarlos, y qué lugar ocupan estos agentes dentro de la trama de relaciones de poder de cada caso protagonizado por un joven. Esto demanda prestar atención a cuáles son las posibilidades de intervención con las que cuentan las profesionales de la OMAS para determinar cuándo un joven ha cumplido con cierto "acontecimiento significativo", en tanto punto de referencia temporal, que pueda definir el paso a otra "etapa" en la recuperación de esa persona. Iluminar esta potestad institucional, desde la concepción foucaultiana del poder en términos de poder/saber (Foucault, 2001) para analizar las relaciones de los aparatos institucionales con los individuos, permite dar cuenta de cómo el ejercicio de este poder se extiende al control del tiempo de las personas aun cuando ellas no se encuentren ya privadas de libertad. Aquí, entonces, el cómputo temporal funciona como instrumento político del Estado, ligado a los conceptos de justicia, ley y capacidad de agencia de los sujetos (Vargas Cetina, 2007).

Como lo hice notar, los márgenes de acción de las trabajadoras sociales para contribuir con determinados procesos subjetivos de un joven se extienden más allá del "mero control y seguimiento". Por el contrario, estas profesionales construyen modos y tipos de autoridad sobre los jóvenes, examinando y evaluando, en sentido foucaultiano, la situación personal de ellos. En estas intervenciones, acontece lo que señala Donzelot (1977), en relación con la construcción de un sujeto tanto como objeto de saber, ya que conocen -con mucha más cercanía que los propios jueces- sus vidas y sus entornos, así como el objeto de intervención. Lo que nos invita a problematizar la definición normativa que designa a estas funciones como de simple "seguimiento y control de medidas".

La tarea asignada, lejos de posicionar a la OMAS como una oficina judicial "meramente administrativa", la ubica en una posición dotada de peso propio dentro de las trayectorias penales juveniles. Esto permite hacer visibles las tensiones inherentes a estos procesos de reforma judicial que oscilan entre la cultura jurídica punitiva y la orientación despenalizadora que impregna a las instituciones creadas bajo la inspiración de la justicia restaurativa, en línea con otros trabajos que estudiaron estas tensiones en el escenario brasileño, como los de Amorim, Kant de Lima y Burgos (2003) y Kant de Lima, Eilbaum y Pires (2009). 
Las trabajadoras sociales de la OMAS contribuyen a la "determinación de los tiempos" (en el sentido antes aludido) de cada joven, y a través de ello logran articular hechos y leyes al modo de sensibilidades legales (Geertz, 1994), al combinar marcos normativos que reconocen formas diferenciadas de contemplar "los tiempos". Uno, el de los "tratamientos tutelares" de la Ley $\mathrm{N}^{\circ} 22.278$ (que no establece plazos precisos, sino que se fijan en función de la "recuperación" del joven); y otro, el de los tiempos procesales de las medidas alternativas que dispone el juez.

Pretendo detenerme aquí, recuperando el planteo inicial, para subrayar cómo la noción de tiempo que hoy impera en este ámbito burocrático se constituye a partir de interacciones y situaciones concretas que van marcando las temporalidades de ese complejo proceso. Entre esas interacciones que involucran a fiscales, defensores y jueces, la labor de las trabajadoras sociales que supervisan y controlan el cumplimiento de las medidas alternativas se construye creativamente modulando los derechos, necesidades y tiempos personales de los sujetos sobre los que se interviene, a la vez que elaborando particulares interpretaciones de los plazos legales que rigen estas medidas. Inmersas en esa tarea, deben lidiar cotidianamente con la superposición de reglas de juego desconectadas entre sí, la ley nacional del régimen penal juvenil que establece ciertas reglas para los tratamientos tutelares y leyes locales de procedimiento penal que fijan modos determinados para la ejecución de las medidas alternativas.

En una entrevista mantenida con Ana, otra trabajadora social de ese organismo, ella afirmaba que los magistrados suelen disponer medidas "insensatas", "fuera de lugar", "medidas superpuestas sin sentido" o "insólitas", es decir, decisiones que bien pueden inscribirse en una lógica de acción institucional que consolida la "supremacía del orden legal por sobre el orden social" (Mello y Baptista, 2011, p. 98). Para ejemplificarlo, me contó que en una causa se dispuso que un joven cumpliera con el reingreso a la escuela primaria, haga tratamiento psicológico, consiga un trabajo estable e iniciara tratamiento de adicciones: "con un gran contrasentido del tiempo: jle piden que haga todo eso en tres meses!" (Registro de diario de campo, entrevista con Ana, Tribunales, Santiago del Estero, marzo de 2018).

Finalmente, se advierte la importancia de prestar atención a las posibilidades concretas -en términos de recursos institucionales- que demanda la evolución de un tratamiento de este tipo. Las reglas de conducta que se imponen solo pueden cumplirse en la medida en que exista una serie de condiciones de posibilidad (la disponibilidad de una oferta educativa inclusiva, la posibilidad efectiva de hacer tratamiento por adicciones en una institución pública, las relaciones conflictivas con las fuerzas policiales en el barrio, por ejemplo) para materializarlas. En la mayoría de los casos, los jóvenes quedan capturados por la demanda institucional que se orienta a que asuman su responsabilidad por el delito denunciado a la vez que no les facilita las herramientas adecuadas para "salir de todo esto", lo que, siguiendo a Haney (2010), ha sido conceptualizado en el trabajo elaborado por, Villalta, Medan y Llobet (2019) como "gobierno por contradicción", para prestar atención a las contradicciones de una agencia estatal que muestra efectos perversos en su faz operativa.

\section{Consideraciones finales}

En nuestro país, las medidas penales alternativas en el ámbito penal juvenil, presentadas como soluciones novedosas dentro de la resolución de conflictos, vienen siendo aplicadas de manera heterogénea y adquieren sentidos diversos en las distintas jurisdicciones provinciales, lo que fue abordado en diferentes trabajos (Medan, 2016; Villalta y Llobet, 2019; Medan y Graziano, 2019; Villalta y Graziano, 2020), así como también en investigaciones elaboradas a demanda de organismos supranacionales (UNICEF, 
2018; BID, 2021). Ahora bien, desde un plano sociológico, el entusiasmo generalizado que las reformas normativas despiertan en los agentes del campo jurídico (Bourdieu y Teubner, 1976) suele traducirse en una sobrestimación de la potencia con la que cuentan los novedosos marcos normativos para transformar la dinámica de un organismo estatal determinado a partir de la incorporación de nuevas reglas de juego y tecnologías de intervención. Estos cambios, en ocasiones presentados como de "vanguardia", tendrían la capacidad de reemplazar a las concepciones, valores y técnicas de intervención ya existentes. Sin embargo, ya Clifford Geertz invitó a pensar estos procesos de manera dinámica e interactiva para dar cuenta de cómo en los procesos de cambio no se produce un "simple paso de lo 'tradicional' a lo 'moderno', sino que se trata de un movimiento espasmódico, retorcido, sin método, que a menudo tiende a recuperar las repudiadas emociones del pasado" (Geertz, 1990, p. 268). Es decir, las reformas no se proyectan sobre un vacío de ideas, sentidos y concepciones, sino que se montan sobre categorías locales y rutinas institucionales de larga duración. Y, particularmente en el ámbito penal juvenil, los cambios propuestos se traman con construcciones de sentidos sobre la peligrosidad/anormalidad de los jóvenes y sus familias. Esto involucra que los procesos de reforma siempre estén sujetos a cambios, ajustes y reacomodamientos institucionales en tanto son objeto de disputa y ocasionan resistencias en su implementación.

En este trabajo, el análisis de los usos concretos que reciben estas medidas a nivel local permitió llamar la atención sobre un particular modo de reacomodamiento institucional en este campo estatal de administración de conflictos (Sinhoretto, 2009) e indagar en las especificidades que adquiere la administración institucional de la conflictividad penal juvenil. Esta reconfiguración de la burocracia judicial está marcada por dos procesos, que son presentados por los actores institucionales entrevistados como insertos en tendencias que operan en sentido diverso. Por una parte, por el mantenimiento de un justicia penal de adultos que tiene competencia penal juvenil. Es decir que aún no existe a nivel local una justicia penal juvenil como fuero especializado, con lo cual la función estatal de intervención como "garante de derechos" se ve en parte menguada por la actuación de jueces penales de adultos que no conocen de las trayectorias juveniles previas a los procesos penales ni tampoco controlan y supervisan el cumplimiento de las medidas que disponen sobre los jóvenes cuando se trata de delitos "contra la propiedad" y otras modalidades delictivas que prevén sanciones menores. Por el otro, la reforma procesal penal a nivel local ha sido presentada como un gran proceso de "modernización" del funcionamiento del sistema penal y ha impulsado entre sus rediseños institucionales a la constitución de la OMAS, con una fuerte carga discursiva orientada hacia la resolución de conflictos en clave restaurativa. En algún punto, dentro de este diseño institucional, las intervenciones de las trabajadoras sociales de esta oficina recuperan esa clave "protectoria" del fuero penal juvenil procurando tomar conocimiento e intervenir sobre las tramas sociales y los entornos familiares/ comunitarios en los que se inscribe la ejecución de las medidas "alternativas" que se imponen a los jóvenes.

La perspectiva adoptada permite tomar distancia de una visión normativa/abstracta que centra su atención en la "incorporación de los estándares internacionales de derechos humanos en materia penal juvenil" a la normativa local, así como en la importancia de la reingeniería institucional, sin detener la mirada en la cotidianeidad del funcionamiento de estas instituciones. Desde este enfoque, considerar los procesos de reforma en términos de "sensibilidades legales" (Geertz, 1994) posibilita indagar en la relación existente entre los hechos y las leyes en este escenario en particular. Y a partir de esto, explorar los sentidos que adquiere esta relación desde las perspectivas de actores acostumbrados a lidiar con los desafíos que engendra la aplicación de medidas alternativas sobre jóvenes y, además, evidenciar cómo los agentes institucionales significan y se 
relacionan con las exigencias normativas así como con las expectativas institucionales que se suscitan en la implementación de estas medidas.

Este análisis partió de la premisa de considerar que la administración judicial no se limita a un campo meramente legal y técnico, en el que sea factible que una reforma procesal pueda sustituir de modo automático un modelo de intervención judicial por otro, sino que, antes bien, estos procesos se insertan con valores morales y concepciones que moldean rutinas institucionales de larga data (Villalta y Graziano, 2020), así como con sensibilidades legales que enlazan hechos y leyes en maneras específicas de administrar justicia sobre jóvenes.

A partir del recurso expositivo de recuperar situaciones etnográficas que den cuenta de microprácticas de la agencia institucional, el argumento central de esta exploración apuntó a revelar cómo estas medidas se recuestan sobre los mismos tópicos que dieron sustento a las medidas tutelares tradicionales: los jóvenes deben recuperar la escolaridad, presentarse periódicamente a tribunales y dar comienzo al tratamiento por adicciones. La reconstrucción de estas situaciones etnográficas hizo posible explorar las perspectivas de jóvenes y actores institucionales y, en particular, permitió indagar en las temporalidades que estructuran las posibilidades de cumplimiento de las medidas por parte de los jóvenes. En cuanto tales, fueron guiadas por el objetivo de iluminar tanto dimensiones estructurales como aspectos cotidianos del funcionamiento de la agencia judicial estudiada, buscando elaborar algunas conclusiones generales sobre los usos contextuales que reciben estas medidas en el orden local.

\section{Financiamiento}

Este trabajo estuvo financiado por el Consejo Nacional de Investigaciones Científicas y Técnicas (CONICET)

\section{Agradecimientos}

Quiero agradecer a Carla Villalta por la imprescindible orientación para hacer esta investigación.

\section{Biografía}

Doctor en Humanidades (UNT). Becario Postdoctoral de CONICET. Docente Facultad de Humanidades Ciencias Sociales y de la Salud (UNSE). 


\section{Q Referencias bibliográficas}

"Abrams, P. (2000). Notas sobre la dificultad para estudiar el Estado. Revista de Antropología y Sociología Virajes, 2(2), 79-98.

» Amorim, M. S., Kant de Lima, R. y Burgos, M. B. (2003). A Administração da Violência Cotidiana No Brasil: A Experiência dos Juizados Especiais Criminais. En Amorim, M. S., Kant de Lima, R. y Burgos, M. B. (Orgs.), Juizados Especiais Criminais, Sistema Judicial e Sociedade no Brasil (pp. 19-52). Niterói: Intertexto.

»Auyero, J. (2012). Pacientes del Estado. Buenos Aires: Eudeba.

»Bourdieu, P. y Teubner, G. (1976). La fuerza del derecho. Elementos para una sociología del campo jurídico. México DF: Siglo del Hombre.

»Das, V. y Poole D. (2008). El estado y sus márgenes. Etnografías comparadas. Cuadernos de Antropología Social, 27, 19-52.

»Donzelot, J. (1977). La policía de las familias. Valencia: Pretextos.

" Eilbaum, L. (2017). Prólogo. En F. Graziano. Pequeños juicios. Moralidades y derechos en la administración judicial para "menores" en la ciudad de Buenos Aires, (pp. 11-13). Buenos Aires: Antropofagia.

"Foucault, M. (2001). La verdad y las formas jurídicas. Barcelona: Gedisa.

» Garapon, A. (1997). Bem julgar. Ensaio sobre o ritual do Judiciário. Traducción de Pedro Filipe Henriques. Lisboa: Instituto Piaget.

»Garland, D. (2018). Castigar y asistir. Una historia de las estrategias penales y sociales del siglo XX. Buenos Aires: Siglo XXI.

» Geertz, C. (1990). La Interpretación de las Culturas. Barcelona: Gedisa.

» Geertz, C. (1994). Conocimiento local: hecho y ley en la perspectiva comparada. En Conocimiento local. Ensayos sobre la interpretación de las culturas (pp. 195-262). Barcelona: Paidós.

»Godoy, M. (2016). Delito, conflicto: sensibilidades legales y trama institucional en el campo de la mediación penal en Salta-Argentina. Revista del Museo de Antropología, 9, 57-68.

» Graziano, F. (2017). Pequeños juicios. Moralidades y derechos en la administración judicial para menores en la ciudad de Buenos Aires. Buenos Aires: Antropofagia.

"Haney, L. (2010). Offending women. Power, punishment and the regulation of desire. Berkeley: University of California Press.

» Kant de Lima, R., Eilbaum, L. y Pires, L. (2009). Construcción de verdad y administración de conflictos en Río de Janeiro: Una mirada sobre las reformas de las instituciones judiciales y policiales. Revista Ava, 16, 33-59.

» Lugones, M. G. (2012). Obrando en autos, obrando en vidas: formas y fórmulas de la protección judicial en los Tribunales Prevencionales de Menores en Córdoba, Argentina, a comienzos del siglo XXI. Río de Janeiro: E-Papers.

"Matta, J. P. y Godoy, M. I. (2016). El movimiento de Mediación en Argentina: procesos, tensiones y afirmaciones. Revista de Estudos e Pesquisas sobre as Américas, 10, 1-23.

" Medan, M. (2016). Justicia restaurativa y mediación penal con jóvenes: una experiencia en San Martín, Buenos Aires. Delito y Sociedad, 1(41), pp. 77-106. 
» Medan, M. (2019) El territorio, la comunidad y la autonomía: ¿discursos mitológicos en los programas sociales destinados a jóvenes en riesgo? Ciudadanías. Revista de políticas sociales urbanas, 4, 139-170.

» Medan, M. y Graziano, F. (2019). Transformaciones, innovaciones y tensiones en la justicia penal juvenil. En V. Llobet y C. Villalta (Coords.). De la desjudicializacion a la refundación de los derechos: transformaciones en las disputas por los derechos de los niños y las niñas (2005-2015), (pp. 449-481). Buenos Aires: TeseoPress.

» Medina, F. (2021). Un libro sobre la vida de los chicos del barrio. Reflexiones metodológicas de una investigación etnográfica. Revista Latinoamericana de Metodología de la Investigación Social-ReLMIS, 16 (en prensa).

» Mello, K. S. S. y Baptista, B. G. L. (2011). Mediação e conciliação no Judiciário: Dilemas e significados. DILEMAS: Revista de Estudos de Conflito e Controle Social, 4(1), 97-122.

» Nader, L. (1994). Harmonia coerciva: a economia política dos modelos jurídicos. Revista Brasileira de Ciências Sociais, 26, 18-29.

» Schuch, P. (2008). Tecnologias da não-violência e modernização da justiça no Brasil. Revista Civitas, 8(3), 498-520.

» Sinhoretto, J. (2009). Campo estatal de administração de conflitos: múltiplas intensidades da justica. Anuario Antropológico, 2, 109-123.

»Tedesco, G. (2017). Tiempos, ritmos y prácticas en institutos para “jóvenes en conflicto con la ley penal”. Prácticas de oficio, 1(19), 69-75.

» Tenenbaum, G. (2018). Violencia juvenil, familias y calles. ¿Dónde se “rescatan” los adolescentes de Montevideo en conflicto con la ley? Revista de Ciencias Sociales, DS-FCS, $31(42), 151-175$.

» Tiscornia, S. (2008). El activismo de los derechos y burocracias estatales. El caso Walter Bulacio. Buenos Aires: Editores del Puerto.

»Vargas Cetina, G. (2007). Tiempo y poder: la antropología del tiempo. Revista Nueva Antropología, XX(067), 41-64.

»Villalta, C. (2013). Un campo de investigación: las técnicas de gestión y los dispositivos jurídico-burocráticos destinados a la infancia pobre en la Argentina. Revista Civitas, 13(2), 245-268.

»Villalta, C., Graziano, F., Gesteira, S., Grinberg, J., Fernández Tuñón, C., Pérez Álvarez, J. (2017. Septiembre). Burocracias, derechos y nuevos procedimientos: las medidas alternativas en la justicia penal juvenil. En VI Jornadas de Antropología Social del Centro. Universidad Nacional del Centro de la Provincia de Buenos Aires y el NuRES (Núcleo Regional de Estudios Socioculturales), Olavarría, Argentina.

» Villalta, C., Medan, M. y Llobet, V. (2019). Entre inercias burocráticas y evaluaciones sobre las familias: adolescentes privados de libertad en Buenos Aires, Argentina. Estudios Socio-Jurídicos, 21(1), 293-326.

»Villalta, C. y Llobet, V. (Coords.) (2019). Justicia juvenil restaurativa. Los diversos usos de las medidas alternativas al proceso penal en la Argentina. En De la desjudicializacion a la refundación de los derechos: transformaciones en las disputas por los derechos de los niños y las niñas (2005-2015), (pp. 383-409). Buenos Aires: Teseo Press.

»Villalta, C. y Graziano, F. (2020). Justicia restaurativa y medidas alternativas al proceso penal en la Argentina. Transformaciones y disputas en la justicia penal para adolescentes. Revista nuestrAmérica, 8(15), 57-73. 


\section{Otras fuentes consultadas}

» Reglamento Oficina de Control de Medidas Alternativas y Sustitutivas de Santiago del Estero.

» Informe Justicia Juvenil. Investigación sobre medidas no privativas de la libertad y alternativas al proceso judicial en Argentina, elaborado por UNICEF, septiembre de 2018.

" Informe Consultoría para la elaboración de estudios sobre el sistema penal juvenil, elaborado por Banco Interamericano de Desarrollo (BID) durante el año 2019, en prensa. 